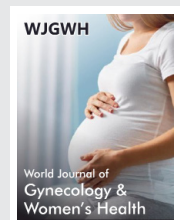

\title{
Chromosome 16 Mutations and Congenital Genitourinary Malformations
}

\author{
Daniel Faustin, Morgan Barlow*, Anuja Trivedi and Naila Ferris \\ Wyckoff Heights Medical Center, USA
}

*Corresponding author: Morgan Barlow, Wyckoff Heights Medical Center,

Department of OB/Gyn, USA

\author{
Received Date: November 13, 2018 \\ Published Date: November 20, 2018
}

\section{Introduction}

Posterior urethral valve (PUV) disorder is an obstructive anomaly of the genitourinary tract. Found only in males, this condition is caused by an obstructing membrane in the posterior valve of the urethra. During the early stages of embryogenesis, the most caudal end of the wolffian duct is absorbed into the primitive cloaca, forming the posterior urethral folds [1]. In fetuses with PUV, the development of these structures is arrested resulting in fusion of these primitive folds, leading to an obstruction of the outflow of urine. This pathologic finding presents on a spectrum of varying degrees, wherein the obstruction may cause only mild symptoms and present as late as early infancy or cause severe and irreversible damage during early fetal development. In this case, we investigate the possible association between chromosome 16 and the development of the renal system, and the potential for congenital malformation in the setting of genetic alteration.

\section{Case}

We describe a 30-year-old G1P0 with a fetus that has multiple congenital anomalies and a chromosome 16 duplication at 16q24.2. This patient initially presented to our institution at 12 weeks gestation without any obstetrical complaints. She had no past medical or surgical history and no family history of congenital or chromosomal abnormalities. Initial sonogram revealed a viable fetus. Prenatal labs were found to be within normal limits, aside from a positive quantiferon gold.

Fetal anatomy survey performed at 20 weeks gestation revealed multiple fetal anomalies, including bilateral cystic dilation of the pelvic and calyceal spaces, bilateral hydronephrosis, an enlarged urinary bladder, and oligohydramnios. Several sonographic images suggested a dilated posterior urethra, demonstrated by the pathognomonic "keyhole appearance". PUV was suspected at this time and close follow-up was recommended. The patient was evaluated again at 22 weeks gestation, and a targeted fetal ultrasound revealed worsening oligohydramnios with persistent

distention of the urinary system. These findings confirmed the suspicion of posterior urethral valve disorder.

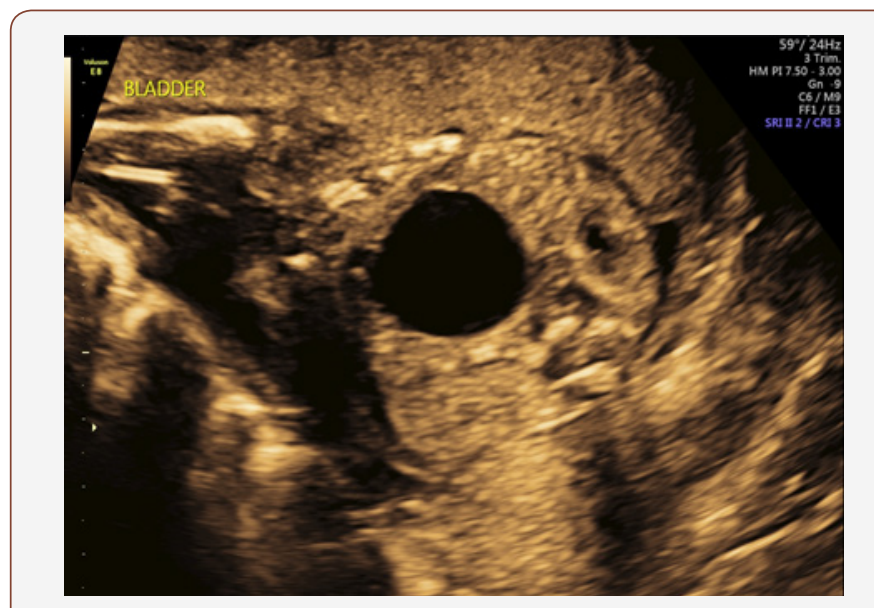

Figure 1: Fetal bladder with "keyhole" appearance.

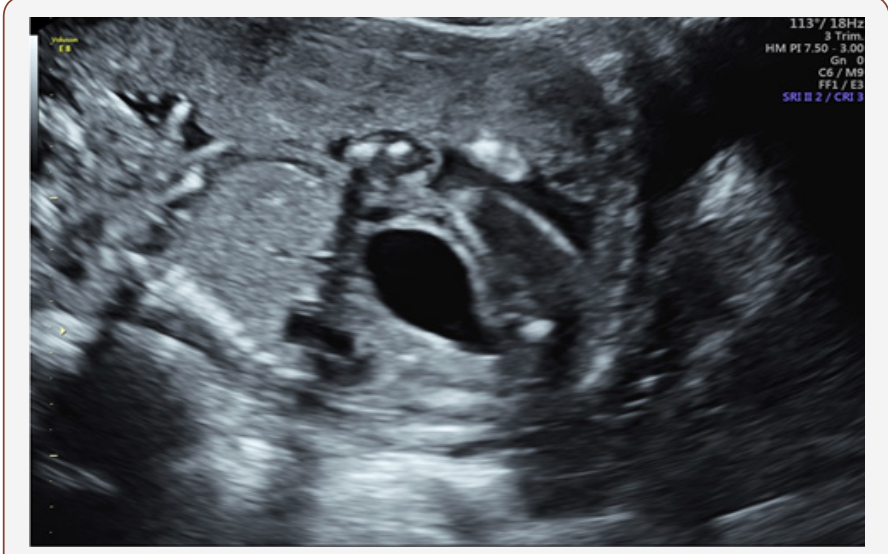

Figure 2: Fetal bladder with "keyhole" appearance, alternate view.

The patient was counseled on the findings and expectations of this anomaly during the anatomy scan and genetic testing was offered. Amniocentesis showed elevated AFP (23.1 mcg/mL) 
and chromosomal analysis revealed a 46XY fetus. Additionally, the chromosomal micro assay exhibited a fetal chromosomal abnormality: arr [hg19] 16q24.2 (88, 059, 561-88, 647, 325) x 3. Approximately $588 \mathrm{~kb}$ interstitial gain of $16 \mathrm{q} 24.2$ was detected in this male fetus. This duplication included a segment of the BANP gene (OMIM: 611564), complete copies of the genes ZNF469 (612078), ZFPM1 (601950) and MIR5189, plus a segment of the ZC3H18 gene (not annotated in OMIM) (Figures 1-5).

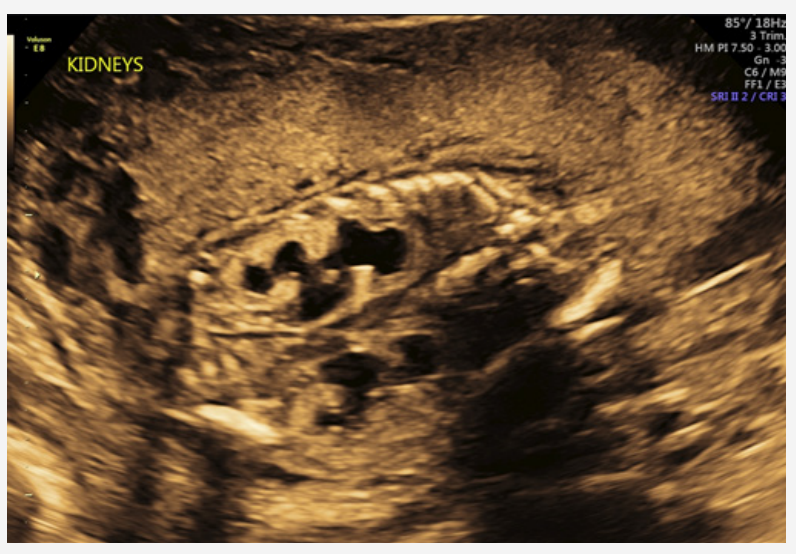

Figure 3: Bilateral dilation of the pelvic and calyceal spaces on fetal ultrasound.

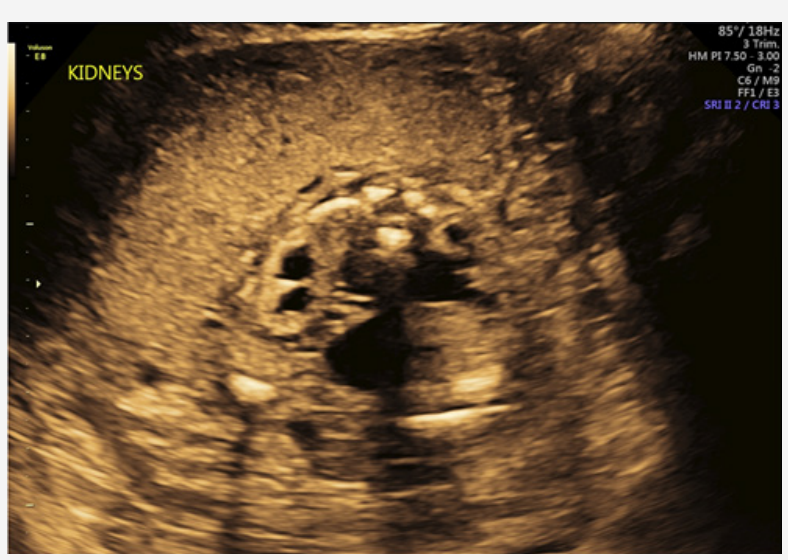

Figure 4: Transverse view of the kidneys with dilated pelvic and calyceal spaces.

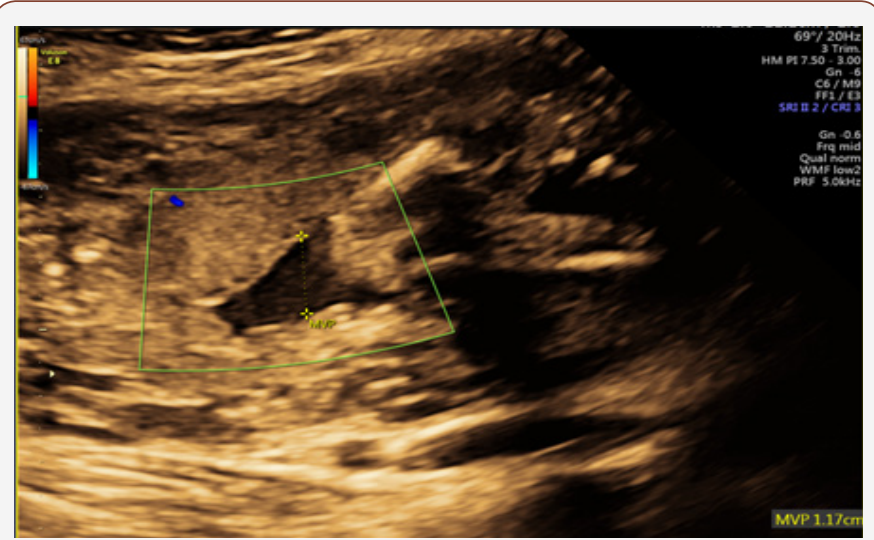

Figure 5: Oligohydramnios with MVP $<2 \mathrm{~cm}$

The patient was referred to a tertiary center for possible urethral stent placement in utero. The institution she was referred to felt as though she was not a candidate for this intervention secondary to the suspected irreversible damage to the renal parenchyma. Because of this poor prognosis, the patient opted to terminate the pregnancy at 23 weeks gestation. The patient was referred to another institution for termination of pregnancy. The patient and her partner were followed closely in the post-abortive period. Karyotyping with chromosomal analysis was recommended to discriminate between a familial variant and a de novo genetic alteration.

The mother of this fetus opted to perform individual microarray testing at a tertiary center. The results yielded no chromosomal abnormality in the mother.

\section{Discussion}

Posterior urethral valve disease was identified and confirmed in this case, in addition to a duplication at 16q24.2. With no known familial congenital malformations, genetic disorders, or consanguinity, the question remains whether this duplication occurred de novo or was inherited, and whether this genetic variant is causal of the urinary anomaly. The urinary tract anomaly in this case, in conjunction with other cases noted in the literature, suggest the possibility of involvement of chromosome 16 in the development of the urinary system.

Mutations on chromosome 16 typically result in spontaneous abortion or have arrested development in early pregnancy [2]. Fetuses with this mutation that have survived gestation are mosaics [1]. These mosaics, however, are typically found to have renal and urinary malformations of varying degrees, dysmorphic features, intellectual disability, and/or autism of varying degrees. The degrees of these clinical features correlate to the size of the microdeletion or duplication; the larger the mutation, the more severe the clinical presentation. Micro assay determined that this fetus had a chromosomal duplication at 16q24.2 with genes BANP, ZNF469, ZFPM1, MIR5189 and ZC3H18 being affected. There have been no associations made between a duplication at this locus and urinary malformations. However, there have been other documented cases of chromosome 16 mutations associated with renal and urinary malformations. These mutations include microdeletions in 16q24.1q24.2, 16p11.2, deletions of 16q24.2q24.3 and 16q24.3, duplication of 16p13.11 and 16p11.2.

There are a few documented cases of haploinsufficiency of the chromosome 16q24 genes being associated with congenital renal malformation. Renal malformations were reported by Novara, et al. [3], who described a deletion of 16q24.1q24.2, and Handrigan, et al. [4], who reported a deletion of 16q24.2. In both studies, a possible relationship was proposed between the minimal overlap of the genes FBX031, MAP1LC3B and ZCCHC14 with the finding of renal malformation $[3,4]$. Additionally, the microdeletions and microduplications of the ANKRD11 gene, which have resulted in haploinsufficiency, are associated with an augmented incidence of renal malformations. It has been proposed that mutations in this region may play a critical role in the development of the renal system; thus, variations in these regions may lead to genitourinary anomalies.

Urinary tract malformations have also been reported in siblings born to consanguineous parents, with a duplication at 16p13.11 
[5]. In a case reported by Houcinat, et al. [5], two male children were born to a father with a known history of unilateral renal agenesis. Both children were found to have urinary malformations and a duplication at $16 \mathrm{p} 13.11$. One child was noted to have posterior urethral valves with chronic renal disease and the other had mild chronic renal disease secondary to ureteropelvic junction obstruction. This study suggests that chromosome 16 mutations may be inherited. However, the parents in our case are nonconsanguineous and both parents have denied any family history of urinary malformations, intellectual disabilities, or congenital abnormalities. It is likely that the malformations noted in this fetus are linked to a chromosomal abnormality that is present only in the fetus (de novo). In fact, one cohort study reviewed 12 patients with known deletions on chromosome 16 at 16q24.2q24.3.5, and 11 were noted to be de novo. The remaining one case had a deletion of unknown origin. (The probability of this micro-mutation occurring in two separate individuals in a non-consanguineous relationship is unlikely.)

Whether the malformation in this case was the result of inherited genetic variants, or occurred de novo, relies on the chromosomal analysis of the father. Regardless of the etiology, there have been multiple cases reported describing chromosome 16 mutations and congenital renal abnormalities. We postulate that there is a link between mutations on chromosome 16 and urinary malformations.

\section{Acknowledgement}

None.

\section{Conflict of Interest}

No Conflict of Interest.

\section{References}

1. Bomalaski MD (2018) Posterior urethral valves. Background, pathophysiology, etiology.

2. Weremowicz S, (2018) Congenital cytogenetic abnormalities.

3. Novara F, Rinaldi B, Sisodiya SM, Coppola A, Giglio S, et al. (2017) Haploinsufficiency for ANKRD11-flanking genes makes the difference between KBG and 16q24.3 microdeletion syndromes: 12 new cases. Eur J Hum Genet 25(6): 694-701.

4. Handrigan GR, Chitayat D, Lionel AC, Pinsk M, Vaags AK, et al. (2013) Deletions in 16q24.2 are associated with autism spectrum disorder, intellectual disability and congenital renal malformation. J Med Genet 50(3): 163-173.

5. Houcinat N, Llanas B, Moutton S, Toutain J, Cailley D, et al. (2015) Homozygous 16p13.11 duplication associated with mild intellectual disability and urinary tract malformations in two siblings born from consanguineous parents. Am J Med Genet Part 167A(11): 2714-2719. 